\title{
The Power and the Puzzles of Auditory Interfaces
}

\author{
Penelope M. Sanderson \\ The University of Queensland \\ St Lucia, Queensland, Australia 4072 \\ psandersonaitee.uq.edu.au
}

\begin{abstract}
Auditory interfaces are increasingly prevalent in work and everyday environments. I survey recent uses of non-speech auditory interfaces and advances in knowledge about them, highlighting research from The University of Queensland.
\end{abstract}

Keywords: Auditory interface design, auditory displays, distraction, multimodal displays, multisensory integration, individual differences, music.

\section{Overview}

Auditory interfaces are present in work and everyday environments as diverse as stockmarket trading, web traffic monitoring, patient monitoring, phones and personal assistants, vehicles, defence contexts, and assistive technologies for the visually impaired-I provide examples of recent designs. For the last 20 years in particular, auditory interface design has been stimulated by a multidisciplinary community drawn from computer science, psychology, physics, music, performance, acoustics, and other areas. During that time there has been greater clarity in how to design different forms of auditory interfaces, such as audifications, parameter-mapping sonifications, model-based sonifications, auditory icons, and earcons.

Successful design of auditory interfaces is challenging and requires attention to many issues, some of which are supported with a long theoretical and empirical tradition, and some which are not. Principles of auditory perception and psychoacoustics play a crucial role. Because auditory interfaces can inform or distract, or both, understanding how auditory attention works is also crucial if a display is to work as intended. Auditory interfaces work alongside information from the other senses such as vision, touch, and the vestibular system, so understanding how multimodal information is processed is increasingly important. Because auditory information is naturally heard by all within a certain range, understanding factors underlying people's tolerance of auditory displays is important, as is determining the appropriate scope of social participation in auditory information. Finally, individual differences can affect how well people can use auditory interfaces, yet such differences are seldom tested

In the address I will provide examples from our research on auditory displays that address some of the above questions, supplemented with work from other laboratories. Our main focus at The University of Queensland has been displays for medical 
contexts, but the principles we have uncovered extend well beyond healthcare. In one branch of our work we have addressed the problem of confusion between alarms. Recently we performed a controlled study of some controversial alarm sounds proposed by Patterson and Edworthy over 20 years ago-the results have important lessons for designers of auditory interfaces. In another branch of our work we have explored the question of how people combine auditory information with visual information from a head-mounted display while they are walking around and performing physical tasks. The results suggest that there is a subtle interplay of vestibular, optic, haptic, and auditory factors, and leave open many puzzles still to solve.

In much of our research, individual differences figure quite strongly. For example, we often find distinct performance differences between listeners with and without musical training, yet research and evaluations of auditory interfaces seldom classify participants in terms of musicality or music training. We have also uncovered differences in how easy and useful listeners at different points on Eyesenck's emphathy/psychoticism scale find it to work with auditory information. If such findings prove to be generalisable, they may have important implications for design of auditory interfaces and for how we deliver auditory information to listeners. 